\title{
Caspases and apoptosis - biology and terminology
}

\author{
David L. Vaux ${ }^{\star, 1}$ \\ 1 The Walter and Eliza Hall Institute, Post Office Royal Melbourne Hospital, \\ Victoria 3050, Australia \\ * corresponding author: tel: (+) 6139345 2544; fax: (+) 6139347 0852; \\ e-mail: vaux@wehi.edu.au
}

This edition of Cell Death and Differentiation contains a letter ${ }^{1}$ and three reviews ${ }^{2-4}$ that raise issues of the biology and terminology of cell death. Each addresses a fundamental question in cell death research: Is apoptosis a caspase mediated process of cell death, or can apoptosis be implemented by other mechanisms? From reading these pieces it will be clear that the answer will not only depend on the underlying biology, but also on the precise meaning of the terms used: as the word 'apoptosis' means different things to different people, the same question may have several mutually exclusive, but correct answers. One hopes that these conflicts may help stimulate adoption of a more precise terminology, more detailed description of experiments, and new experiments that reveal the nature of the mechanisms of cell death, which is, after all, what we are really interested in.

To introduce the letter and the reviews, I will start from a point that all can agree upon: every cell that is living can be killed, therefore there is such a thing as 'non-physiological' cell death. This kind of cell death occurs when some process that is essential for suvival is interrupted.

It is also clear that the great majority of multicellular organisms (and a number of unicellular ones) at one time or another cause some of their own cells to die. These cell deaths, which may be cell autonomous (suicidal) or noncell autonomous (murderous) can be thought of as 'physiological cell deaths' in the sence that they can be observed when there is no evidence of a noxious agent. The main goal of the cell death field is to determine the molecular nature(s) and regulation of these physiological cell deaths, whether they occur in prokaryotes or eukaryotes, single-celled or multicellular organisms, plants or animals.

Unfortunately the term 'physiological cell death' is not perfect, as there are examples of cells activating a 'physiological' death mechanism in response to a 'pathological' stimulus, to commit suicide before they are killed. Indeed, some of Kerr's earliest observations of 'shrinkage necrosis' (later termed apoptosis) were in livers exposed to hepatotoxins. 5

The term 'programmed cell death' is problematical because it is used differently by different investigators. It has been used to refer specifically to developmental deaths, because development (especially of invertebrates) is 'programmed', 7 it has been used to refer to one or more specific intracellular molecular 'programs' of cell death, and it has been used to refer to all cell deaths that are not caused by noxious agents.

'Apoptosis' is the most recent and most popular term, but is in some ways the least well defined. Originally apoptosis was defined purely on morphological grounds, ${ }^{8}$ it has therefore been acceptable to call 'apoptosis' anything that looks like apoptosis. Currently 'apoptosis' may have many other meanings. In a figure, for example, a y axis labeled 'apoptosis' might refer to cells with a particular nuclear or cytoplasmic morphology, cells with degraded DNA, cells with a leaky plasma membrane, cells that bind annexin, cells with activated caspases, or cells with cleaved keratin 18.

In their letter, Drs Samali, Zhivotovsky, Jones, Nagata and Orrenius have proposed that the term 'apoptosis' be redefined as 'caspase-mediated cell death with associated apoptotic morphology.' 1 There are two chief merits to this proposal. First, if there is an agreed terminology, when someone refers to 'apoptosis' others would know precisely what is meant. Secondly, such a definition would encourage researchers to look beyond the appearances of cell death, and focus on the underlying biochemical mechanisms and would naturally lead to the next question: what activated the caspases that caused the cell to die?

The word 'apoptosis' has already had a difficult history it has even been suggested that it was used as a deliberate attempt by British scientists to establish barriers between them and others. ${ }^{9}$ If we are going to re-define apoptosis in terms of caspase activation, then should we go even further, and retire the term 'apoptosis' altogether? After all, the term 'programmed cell death' is older, ${ }^{7}$ and even though the concept of apoptosis tied together cell suicide in physiological, pathological and developmental contexts, ${ }^{8}$ it is quite possible our understanding of the process would have been just as good had it never been used. As Clarke and Clarke has pointed out, ${ }^{10}$ physiological cell death was discovered independently seven times prior to the turn of the century. The reason it was forgotten at earlier times but not with its rediscovery in 1972 may simply reflect the fact that on this occasion it was followed by discoveries of the nature of the underlying mechanisms. In fact, none of the early papers describing cell death during development of $C$. elegans (i.e. the ced-mutant worms), ${ }^{11-13}$ or the control of mammalian cell death (i.e. the ability of $\mathrm{Bcl}-2$ to prevent mammalian cell death) ${ }^{14}$ mentioned the word apoptosis, and the surge in interest in cell death did not come with the adoption of the term in 1972, but with the identification of components of the cell death mechanism that began 16 years later.

However, there are some problems with the proposal put by Samali et al. ${ }^{1}$ The first definition they have proposed for apoptosis, as 'a caspase-mediated cell death with associated apoptotic morphology' is circular, because 
'apoptotic' appears twice. Adopting such a definition would require that both caspase activation and the appropriate morphology to be demonstrated before it would be certain that 'apoptosis' was occurring. Determination of caspase activation alone would be inadequate, because, as the authors point out, some caspases are involved in cytokine activation, rather than cell death. If we determine what is apoptosis on the basis of morphology alone, we are back to where we started.

Secondly, there is the question of caspase-independent apoptosis, an issue of biochemistry rather than of terminology, which brings me to the three accompanying reviews.

These reviews ${ }^{2-4}$ all discuss circumstances in which mammalian cells die, either with or without classical apoptotic morphology, in the absence of caspase activity. Alternative mediators and mechanisms of physiological cell death are proposed, including Apoptosis Inducing Factor (AIF), oxygen free radicals, Bax and Bak, and death by autophagic degeneration.

Two questions need to be kept in mind when reading these reviews. First, is this a 'physiological' death process, or could cell death be 'pathological', resulting from interruption to a vital metabolic process? Many investigators will remember the story of Dad-1, which was initially proposed to be an inhibitor of apoptosis, ${ }^{15}$ but turned out to be an oligosaccharyltransferase essential for cell survival.

Second, are the caspase inhibitors used sufficiently potent and specific to conclude that all caspase activity is blocked and no other enzymes are affected? Often, the evidence for caspase-independent cell death is based on observations of cells in which caspase activity has been inhibited by the irreversible tripeptide caspase inhibitor zVAD-fmk, and therefore rely on its ability to block all caspase activity but have no effects on other cellular components. As some caspases may not yet be identified, and as zVAD-fmk is not a particularly good inhibitor of caspase- $2,{ }^{16}$ and as it can affect non-caspase molecules (cathepsin $\mathrm{D}$ for example) $^{17}$ these conclusions may be premature.

Skepticism is also in order because of the genetic experiments in caspase mutants. If mitochondria in every cell of ced-3:ced-9 mutant worm lose their membrane potentials, and leak cytochrome c, AIF, and free radicals, why does it have no detectable effects on cell physiology during development, or for the life of the worm? Perhaps mammals have evolved an additional, non-caspase dependent cell death mechanism. If so, it is curious that it involves phylogenetically old components such as cytochrome $\mathrm{c}$ and AIF.

The way forward is to encourage use of 'apoptosis' to refer to 'caspase-mediated cell death' in order to promote consideration of the underlying biochemistry. However, it is too early to be prescriptive, particularly as challenges to the equivalence of apoptosis and caspase-mediated cell death are still appearing, as illustrated by the three reviews. In a spirit of promoting clear communication and understanding of mechanisms, authors of manuscripts should include on $y$ axes and in figure legends descriptions of the test carried out, whether it is a test of membrane permeability, annexin binding, DNA degradation, chromium release, caspase activation, loss of clonagenicity, chromatin condensation, or substrate cleavage, rather than using jargon terms such 'apoptosis' or even 'cell death'. In this way there will be less misunderstanding, and challenges to the notion that caspases are the key mediators of apoptosis can be evaluated more objectively.

\section{References}

1. Samali A, Zhivotovsky B, Jones D, Nagata S and Orrenius S (1999) Letter. Cell. Death Diff. 6: 495-496

2. Borner $C$ and Monney L (1999) Review. Cell Death Diff. 6: 497-507

3. Kitanaka C and Kuchino Y (1999) Review. Cell Death Diff. 6: 508-515

4. Lorenzo HK, Susin SA, Penninger J and Kroemer G (1999) Review. Cell Death Diff. 6: $516-524$

5. Kerr JFR (1969) An electron microscopic study of liver cell necrosis due to heliotrine. J. Pathol. 97: 557-562

6. Kerr JFR (1970) An electron microscopic study of liver cell necrosis due to alitocin. Pathology 2: 251-259

7. Lockshin R, Williams C (1965) Programmed cell death. II. Endocrine potentiation of the breakdown of the intersegmental muscles of silkworms. J. Insect. Physiol. 11: 803-809

8. Kerr JF, Wyllie AH, Currie AR (1972) Apoptosis: a basic biological phenomenon with wide-ranging implications in tissue kinetics. Br. J. Cancer 26: 239-257

9. Kleine B (1989) Obfuscation. Nature 337: 402

10. Clarke PG and Clarke $S$ (1996) Nineteenth century research on naturally occurring cell death and related phenomena. Anatomy \& Embryology 193: 81 99

11. Hedgecock EM, Sulston JE and Thomson JN (1983) Mutations affecting programmed cell deaths in the nematode Caenorhabditis elegans. Science 220: $1277-1279$

12. Horvitz HR, Ellis HM and Sternberg PW (1982) Programmed cell death in nematode development. Neurosci. Commentaries 1: 56-65

13. Ellis HM and Horvitz HR (1986) Genetic control of programmed cell death in the nematode C. elegans. Cell 44: 817-829

14. Vaux DL, Cory S and Adams JM (1988) Bcl-2 gene promotes haemopoietic cell survival and cooperates with c-myc to immortalize pre-B cells. Nature 335: 440 442

15. Nakashima T, Sekiguchi T, Kuraoka T, Fukushima K, Shibata Y, Komiyama S and Nishimoto T (1993) Molecular cloning of a human cDNA encoding a novel protein, DAD-1, whose defect causes apoptotic cell death in hamster BHK21 cells. Mol. Cell. Biol. 13: 6367-6374

16. Garcia-Calvo M, Peterson EP, Leiting B, Ruel R, Nicholson DW and Thornberry NA (1998) Inhibition of human caspases by peptide-based and macromolecular inhibitors. J. Biol. Chem. 273: 32608-32613

17. Schotte P, Declerq W, Van Huffel S, Vandenabeele P and Beyaert R(1999) Nonspecific effects of methyl ketone peptide inhibitors of caspases. FEBS Lett. 442: $117-121$ 